\title{
Protocatechualdehyde restores endothelial dysfunction in streptozotocin-induced diabetic rats
}

\author{
Bin $\mathrm{Ji}^{1}$, Kaiming Yuan ${ }^{1}$, Jun $\mathrm{Li}^{1}$, Bon Jeong $\mathrm{Ku}^{2}$, Po Sing Leung ${ }^{3}$, Wei He${ }^{1}$ \\ ${ }^{1}$ Department of Anesthesiology and Perioperative Medicine, The Second Affiliated Hospital and Yuying Children's Hospital of Wenzhou Medical \\ University, Key Laboratory of Anesthesiology of Zhejiang Province, Wenzhou Medical University, Wenzhou, China; ${ }^{2}$ Department of Internal \\ Medicine, Chungnam National University College of Medicine, Daejeon, Republic of Korea; ${ }^{3}$ School of Biomedical Sciences, Faculty of Medicine, \\ The Chinese University of Hong Kong, Hong Kong, China \\ Contributions: (I) Conception and design: B Ji, W He; (II) Administrative support: W He; (III) Provision of study materials or patients: B Ji, K Yuan; \\ (IV) Collection and assembly of data: B Ji; (V) Data analysis and interpretation: B Ji, J Li; (VI) Manuscript writing: All authors; (VII) Final approval \\ of manuscript: All authors. \\ Correspondence to: Wei He. Department of Anesthesiology and Perioperative Medicine, The Second Affiliated Hospital and Yuying Children's \\ Hospital of Wenzhou Medical University; Key Laboratory of Anesthesiology of Zhejiang Province, Wenzhou Medical University, Wenzhou 325027, \\ China. Email: jarryhw@126.com.
}

Background: The present study was conducted with the aim of clarifying the effects of protocatechualdehyde (PCA) on the endothelial function in streptozotocin (STZ)-induced diabetic rats.

Methods: Sprague Dawley (SD) rats were intraperitoneally injected with STZ (single dose of $60 \mathrm{mg} / \mathrm{kg}$ ). Diabetic model rats were given PCA ( $25 \mathrm{mg} / \mathrm{kg} /$ day) via gavage feeding for 6 weeks. Vascular function was studied; superoxide anion and nitrotyrosine levels were assessed; and nicotinamide adenine dinucleotide phosphate hydrogen (NADPH) oxidase as well as total superoxide dismutase (SOD) activity were detected. Protein expression of phosphorylated endothelial nitric oxide synthase (P-eNOS), total endothelial nitric oxide synthase (T-eNOS), p22 $2^{\text {phox }}, \mathrm{p} 47^{\text {phox }}$ and Cu/Zn-SOD were measured by Western blot analysis.

Results: PCA treatment significantly ameliorated the impairment of acetylcholine- evoked endotheliumdependent relaxation, with no obvious effects observed on the blood glucose or body weight in the STZinduced diabetic rats. Expression levels of aortic P-eNOS/T-eNOS and endothelial nitric oxide synthase (eNOS) activity were decreased in STZ-induced diabetic rats while they remained unchanged in PCAtreated rats. However, PCA treatment improved oxidative inactivation of nitric oxide (NO) and decreased the levels of superoxide anion and nitrotyrosine in the aorta of STZ-induced diabetic rats; these were achieved by reducing the level of nitrotyrosine and down-regulating $\mathrm{p} 47^{\text {phox }}$ and $\mathrm{p} 22^{\text {phox }}$ expression, as well as up-regulating $\mathrm{Cu} / \mathrm{Zn}$-SOD protein expression. Consistently, the effects observed were associated with a decrease in NADPH oxidase activity and an increase in total SOD activity.

Conclusions: Our results indicate that the administration of PCA may be protective against oxidative stress and may restore endothelial function by improving vascular NO oxidative inactivation in diabetic condition.

Keywords: Diabetes mellitus (DM); protocatechualdehyde (PCA); endothelial function; oxidative stress

Submitted Sep 28, 2020. Accepted for publication Apr 22, 2021.

doi: $10.21037 / \mathrm{atm}-21-1431$

View this article at: http://dx.doi.org/10.21037/atm-21-1431 


\section{Introduction}

Diabetes mellitus (DM), of which hyperglycemia is a distinguishing characteristic, has become a public health concern of global proportions (1). Diabetes-associated cardiovascular complications are major contributors to mortality and morbidity in the diabetic population (2). Meanwhile, endothelial dysfunction, which is generally presented with a decrease in endothelium-mediated vascular relaxation, is encountered early during the development of vasculopathy (3). Endothelial dysfunction is an early and independent predictor of poor prognosis in most forms of cardiovascular disease. Clinical and experimental studies have shown that endothelial dysfunction has critical pathogenic involvement in vascular damage in diabetes (4-7).

Hyperglycemia, diminished production of endotheliumderived relaxing factors nitric oxide (NO) and increased generation of reactive oxygen species (ROS) are the principal causes of endothelium dysfunction in DM $(8,9)$. Protocatechualdehyde (PCA), also known as 3,4-dihydroxybenzaldehyde, is a water-soluble phenolic acid compound that naturally occurs in the herb Salvia miltiorrbiza. In streptozotocin induced diabetic rats, oral administration of PCA for 8 weeks obviously ameliorated the development of diabetic cataract (10). PCA could protect against cerebral ischemia-reperfusion-induced oxidative injury, and the neuroprotective effect involves the protein kinase $\mathrm{C} \varepsilon /$ transcription nuclear factor erythroid 2-related factor $2 /$ heme oxygenase-1 pathway (11). In fact, PCA possesses a variety of pharmacological properties, such as anti-proliferative, anti-inflammatory, and antioxidant activities (10-12), which suggests PCA is a likely pharmacotherapeutic agent in the treatment of diabetes complications. Thus far, data on the in vivo effects of PCA on endothelial dysfunction in DM are lacking. In light of these previous findings and the knowledge gap in the field, we aimed to conduct the present study so as to clarify the effects of PCA on aortic endothelial function in rats with $\mathrm{DM}$ and to find out some related mechanisms. We present the following article in accordance with the ARRIVE reporting checklist (available at http://dx.doi.org/10.21037/ atm-21-1431).

\section{Methods}

\section{Animals}

Male Sprague Dawley (SD) rats, aged 8 weeks (body weight, 200-240 g), were supplied by Shanghai SLAC
Laboratory Animal Co., Ltd. (Shanghai, China), and caged individually under standardized conditions. Experiments were performed under a project license (No.: 201700103) granted by the Wenzhou Medical College Animal Policy and Welfare Committee. All animal experiments complied with the Wenzhou Medical College Guidelines on the Care and Use of Laboratory Animals.

\section{Experimental protocol}

After 1 week of acclimatization, SD rats were intraperitoneally injected with streptozotocin (STZ) (single dose of $60 \mathrm{mg} / \mathrm{kg}$ body weight, freshly dissolved in $5 \mathrm{mM}$ citrate buffer). One week after STZ administration, SD rats with fasting plasma glucose (FPG) $\geq 250 \mathrm{mg} / \mathrm{dL}$ were deemed to be diabetic and were used for subsequent experiments (7). Control and hyperglycemic rats were randomly assigned to 4 groups ( $\mathrm{n}=12$ each group), namely the untreated control group (Con), the PCA-treated Con group (Con + PCA), the untreated STZ-induced diabetic group (STZ), and the PCAtreated STZ group (STZ + PCA). Rats in the two treatment groups were given PCA $(25 \mathrm{mg} / \mathrm{kg} / \mathrm{day}$; Xi'an KinGreen, China) via gavage feeding for 6 weeks. The dosage of PCA was selected based on the dosage used in a previous study (10), and $25 \mathrm{mg} / \mathrm{kg}$ PCA has the sufficient in vivo antioxidant capacity in our preliminary experiment.

After treatment with PCA for 6 weeks, all rats were fasted overnight and anaesthetized. Blood samples were drawn via intracardiac puncture, centrifuged to obtain plasma, and used to assess fasting plasma glucose (FPG). Thoracic aortas from the rats were immediately cut into rings $3 \mathrm{~mm}$ in width for the subsequent vascular function experiments. The remaining aortas were stored at $-80{ }^{\circ} \mathrm{C}$ for future processing. Furthermore, rat carotid artery sections were promptly subjected to freezing in OCT embedding medium (Sakura Finetek, USA) for cryotomy and subsequent detection of superoxide anion.

\section{Vascular function experiments}

The protocols of the present study were described previously $(13,14)$. In brief, aortic rings were suspended between 2 steel hooks in jacketed organ chambers with $10 \mathrm{~mL}$ Krebs solution (mM, $\mathrm{NaCl} 118.5, \mathrm{KCl} 4.7, \mathrm{CaCl}_{2} 1.5$, $\mathrm{MgSO}_{4} 1.2, \mathrm{KH}_{2} \mathrm{PO}_{4} 1.2, \mathrm{NaHCO}_{3} 25.0$, and glucose 11.0) aerated with carbogen and kept at $37^{\circ} \mathrm{C}$ for 1 hour. Force transducers were used to detect isometric tension changes, which were recorded using a polygraph (Nanjing MedEase 
Science and Technology Co., China). After pre-contraction with $10^{-6} \mathrm{M}$ phenylephrine ( $\alpha_{1}$-adrenergic receptor agonist), cumulative relaxation-response curves to $10^{-8}-10^{-4} \mathrm{M}$ acetylcholine and $10^{-10}-10^{-6} \mathrm{M}$ sodium nitroprusside were recorded, respectively. Relaxant responses induced by acetylcholine and sodium nitroprusside were calculated as a percentage of the response to phenylephrine.

Mercaptopropionylglycine (MPG), a powerful superoxide anion scavenger, is able to enhance superoxide anionmediated nitric oxide (NO) oxidative inactivation in aortic rings $(12,15)$. In the present experiment, the response to $10^{-8}$ $10^{-4} \mathrm{M}$ acetylcholine was re-tested in aortic rings previously incubated with $10 \mu M$ MPG. The difference between acetylcholine-induced maximum relaxation values obtained with and without the presence of MPG was taken to represent the superoxide anion-mediated $\mathrm{NO}$ oxidative inactivation.

\section{Biochemical analysis}

FPG was measured by the glucose oxidase method using a commercial kit (Rongsheng Biotech, China). Measurement of total superoxide dismutase (T-SOD) activity in the rat aortas was performed with a commercial kit (Beyotime Institute of Biotech, China) using the xanthine oxidase method as described previously (16). Aortic nicotinamide adenine dinucleotide phosphate hydrogen (NADPH) oxidase activity was measured by using a SOD inhibitable cytochrome $\mathrm{C}$ reduction assay, as described previously $(17,18)$. Vascular endothelial nitric oxide synthase (eNOS) activity was determined using a NOS activity assay kit (Jiancheng Institute of Biotechnology, China) (17).

\section{Determination of vascular superoxide anion}

Cross sections of carotid arteries from the rats were stained with dihydroethidium (DHE, Beyotime Institute of Biotech, China) for evaluation of the in situ superoxide anion levels. The 5 - $\mu \mathrm{m}$ sections were incubated for 30 minutes with $10 \mu \mathrm{M}$ DHE, after which ethidium fluorescent images were obtained using an inverted microscope (Leica Microsystems, Germany) and quantified using image J software.

\section{Determination of vascular nitrotyrosine level}

A commercial enzyme-linked immunosorbent assay (ELISA) kit (LMAI Bio., Shanghai, China) was employed to measure the levels of nitrotyrosine in the aortas of the rats in accordance with the instructions supplied with the kit.

\section{Western blot analysis}

Proteins in the aortic tissue were separated by sodium dodecyl sulfate-polyacrylamide gel and blotted onto polyvinylidene difluoride membranes (Millipore, USA). First, the membranes were blocked, then probed with specific antibodies. The antibodies used were as follows: anti-phosphorylated eNOS (Ser1177, Cell Signaling Technology), anti-eNOS (BD Transduction Laboratories), anti-p22 $2^{\text {phox }}$ (Santa Cruz Biotechnology), anti-p47 $7^{\text {phox }}$ (Santa Cruz Biotechnology), anti-Cu/Zn-SOD (Santa Cruz Biotechnology), and anti-tubulin (Santa Cruz Biotechnology). Antibody binding was detected using an ECL system, and Image J software was used for densitometric analysis.

\section{Statistical analyses}

All data were expressed as mean \pm SD. Between-group differences were tested by analysis of variance (ANOVA) followed by Bonferroni comparison tests. Two-way repeated-measures ANOVA with subsequent Bonferroni comparison tests were used to analyze changes in doseresponse curves over time. $\mathrm{P}<0.05$ was taken to indicate a statistically significant difference.

\section{Results}

\section{Effects of PCA on body weight and FPG}

As indicated in Figure 1A, the body weight of the STZ rats was significantly lower than that in Con rats, and PCA treatment had no effect on body weight. As displayed in Figure $1 B$, the STZ rats displayed elevated FPG compared to the Con rats, and PCA treatment failed to ameliorate this effect.

\section{Effects of PCA on vascular relaxation responses to acetylcholine and sodium nitroprusside}

As shown in Figure $2 A$, the results of the aortic ring experiments revealed that the addition of acetylcholine after pre-contraction with phenylephrine induced relaxation responses in all groups of rats in a concentrationdependent manner. Acetylcholine-evoked endotheliumdependent relaxation was obviously decreased in the STZ rats compared with the Con rats. Treatment with PCA significantly ameliorated the impairment of the endothelial-dependent vasodilator response. Furthermore, 

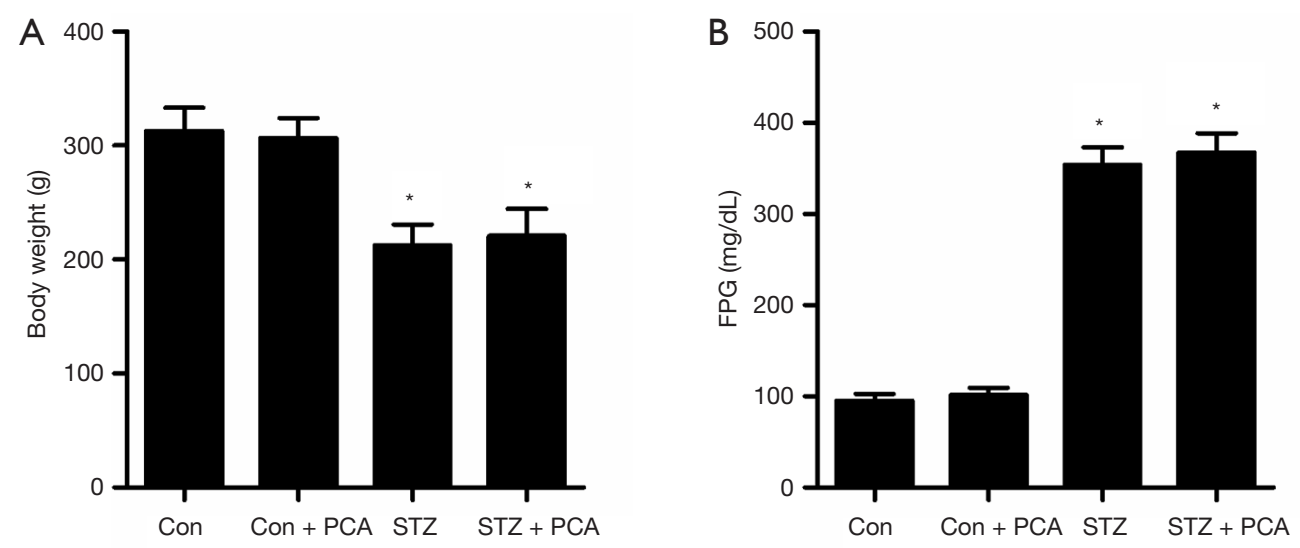

Figure 1 Effects of protocatechualdehyde (PCA) on body weight (A) and fasting plasma glucose (FPG) (B). SD rats were intraperitoneally injected with a single dose of streptozotocin (STZ) $(60 \mathrm{mg} / \mathrm{kg}$ body weight). One week later, rats displaying FPG $\geq 250 \mathrm{mg} / \mathrm{dL}$ were taken to be diabetic rats and were included in subsequent experiments. Control and hyperglycemic rats were randomly assigned to 4 groups ( $\mathrm{n}=12$ each group): the untreated control group (Con), the PCA-treated Con group (Con + PCA), the untreated STZ-induced diabetic group (STZ), the and PCA-treated STZ group (STZ + PCA). Rats in the two treatment groups were administered PCA via gavaging at a dose of $25 \mathrm{mg} / \mathrm{kg} /$ day for 6 weeks. Values are displayed as mean $\pm \mathrm{SD}, \mathrm{n}=12$ per group. ${ }^{*} \mathrm{P}<0.05$ vs. Con rats.
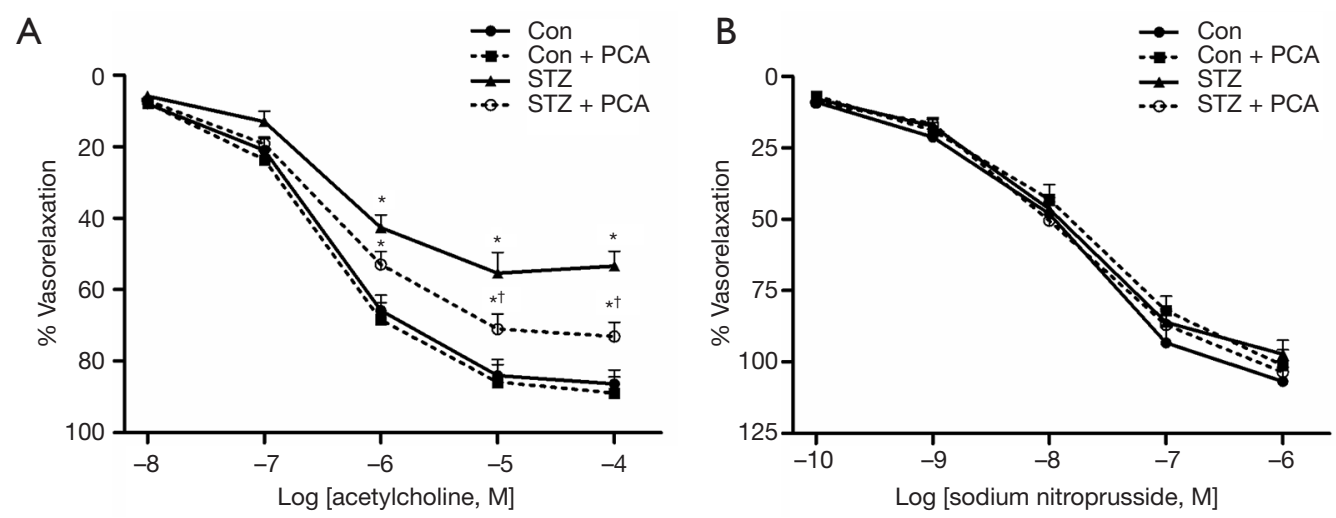

Figure 2 Effects of protocatechualdehyde (PCA) on vascular relaxation responses to acetylcholine (A) and sodium nitroprusside (B). Values are displayed as mean $\pm \mathrm{SD}, \mathrm{n}=5-6$ per group. ${ }^{*} \mathrm{P}<0.05$ vs. Con rats; ${ }^{\dagger} \mathrm{P}<0.05$ vs. streptozotocin $(\mathrm{STZ})$ rats.

no differences were observed between the four groups in terms of endothelium-independent vasorelaxation induced by sodium nitroprusside (Figure 2B).

\section{Effects of PCA on vascular phosphorylated endothelial nitric oxide synthase (P-eNOS) expression and eNOS activity}

As shown in Figure 3, the levels of aortic P-eNOS/total endothelial nitric oxide synthase (T-eNOS) expression and eNOS activity were decreased in the STZ rats compared to the Con rats. Treatment with PCA failed to ameliorate these effects.

\section{Effects of PCA on vascular superoxide anion production, NO oxidative inactivation, and nitrotyrosine levels}

As displayed in Figure 4A,B, superoxide anion production in the carotid arteries of the STZ rats was significantly increased. After treatment with PCA, the superoxide anion content was obviously decreased. MPG, a potent superoxide anion scavenger, improved acetylcholine-induced relaxation in the STZ rats, although it showed no effect on the Con rats. Therefore, the difference between the ACh-induced 

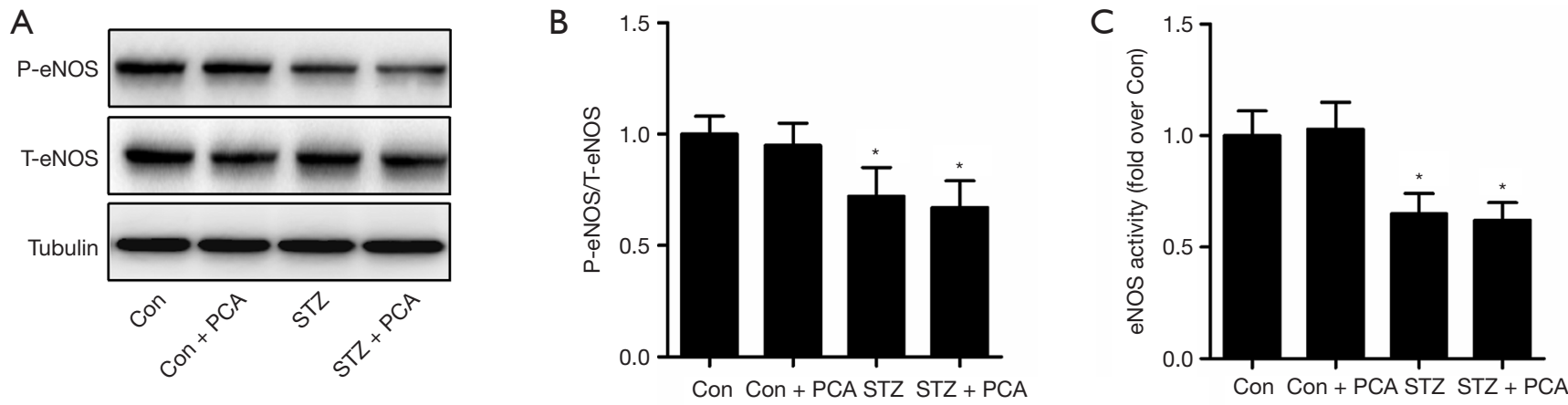

Figure 3 Effects of protocatechualdehyde (PCA) on vascular phosphorylated endothelial nitric oxide synthase (P-eNOS) expression (A and B) and endothelial nitric oxide synthase (eNOS) activity $(\mathrm{C})$. Values are displayed as mean $\pm \mathrm{SD}, \mathrm{n}=4-5$ per group. ${ }^{*} \mathrm{P}<0.05$ vs. Con rats.

A

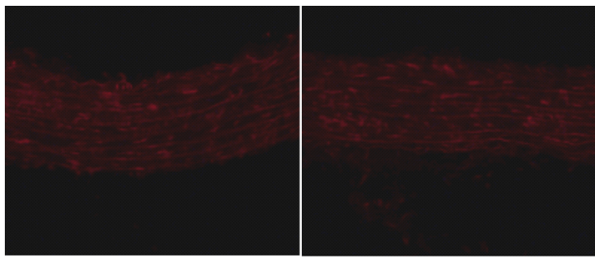

Con

Con + PCA

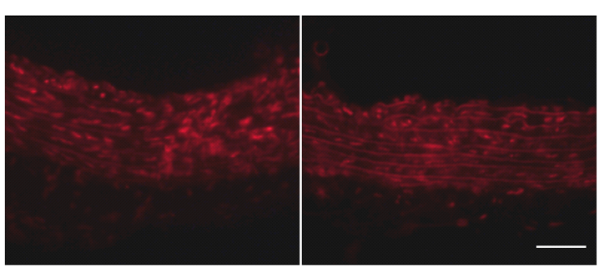

STZ

C

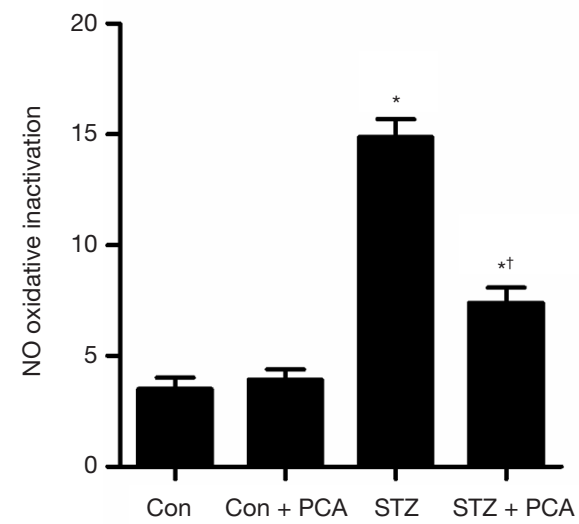

B

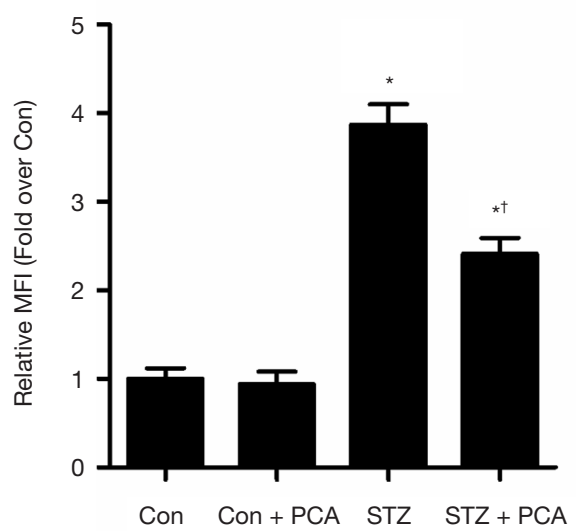

D

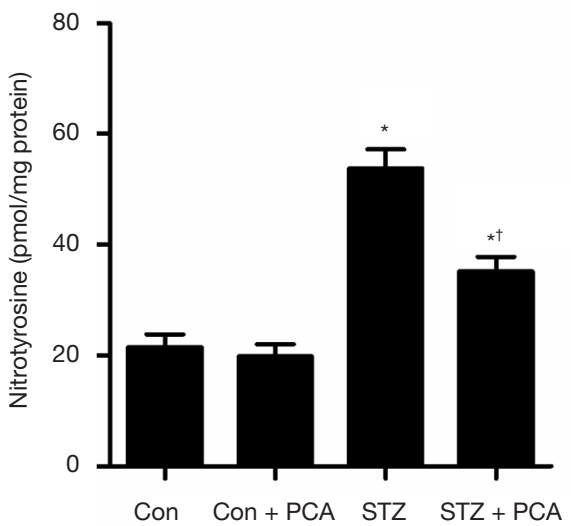

Figure 4 Effects of protocatechualdehyde (PCA) on vascular superoxide anion production, NO oxidative inactivation and nitrotyrosine level. (A) For evaluation of the in situ levels of superoxide anion, rat carotid arteries were stained with dihydroethidium (DHE). Bar $=50 \mu \mathrm{m}$, magnification $\times 200$. (B) The histogram represents the mean fluorescence intensity (MFI) in each group. $n=6$ per group. (C) The difference between acetylcholine-induced maximum relaxation values obtained in the absence and presence of MPG was taken to indicate superoxide anion-mediated nitric oxide (NO) oxidative inactivation. $\mathrm{n}=5-6$ per group (D) Aortic nitrotyrosine level was assessed using ELISA. $\mathrm{n}=6$ per group. Values are displayed as mean $\pm \mathrm{SD},{ }^{*} \mathrm{P}<0.05$ vs. Con rats; ${ }^{\dagger} \mathrm{P}<0.05$ vs. streptozotocin (STZ) rats. 

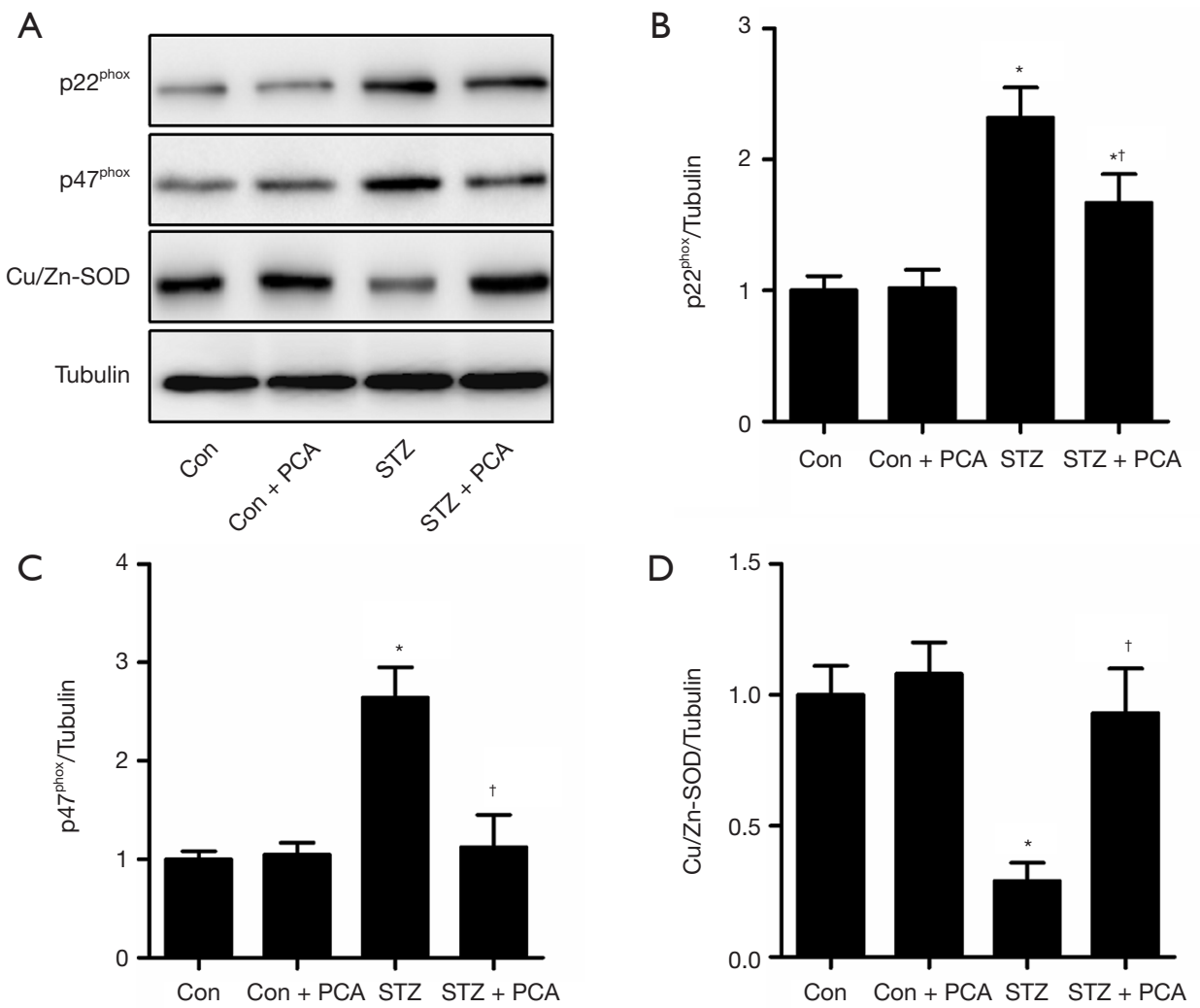

Figure 5 Effects of protocatechualdehyde (PCA) on vascular p22phox, p47phox, and Cu/Zn-superoxide dismutase (SOD) protein expression. Values are displayed as mean $\pm \mathrm{SD}, \mathrm{n}=4-5$ per group. ${ }^{*} \mathrm{P}<0.05$ vs. Con rats; ${ }^{\dagger} \mathrm{P}<0.05$ vs. streptozotocin (STZ) rats.

maximum relaxation values, calculated with and without the presence of MPG, indicated vascular NO oxidative inactivation (Figure $4 C$ ). Treatment with PCA markedly improved NO oxidative inactivation in the aortas of the STZ rats. Furthermore, superoxide anion, produced by NADPH oxidase, reacted with NO to form peroxynitrite, which modifies proteins to form nitrotyrosine. Therefore, nitrotyrosine used as a stable footprint of superoxide anion mediated the inactivation of No. As shown in Figure 4D, the levels of aortic nitrotyrosine in the STZ rats were significantly enhanced compared with those in the Con rats. Treatment with PCA markedly decreased the aortic nitrotyrosine levels.

\section{Effects of PCA on the protein expression of vascular $p 22^{\text {phox }}$, $p 47^{p h o x}$ and $\mathrm{Cu} / \mathrm{Zn}-\mathrm{SOD}$ and the activity of $\mathrm{NADPH}$ oxidase and total SOD}

As shown in Figure 5, the aortic expression of the NADPH oxidase subunits $\mathrm{p} 22^{\text {phox }}$ and $\mathrm{p} 47^{\text {phox }}$ was up-regulated in the STZ rats, compared to that in the Con rats. However,
PCA treatment markedly decreased the protein expression of $\mathrm{p} 22^{\text {phox }}$ and $\mathrm{p} 47^{\text {phox }}$. Furthermore, the activity of vascular NADPH oxidase was significantly higher in the STZ rats, and this increase was obviously ameliorated by PCA treatment (Figure 6A).

The aortic protein expression of $\mathrm{Cu} / \mathrm{Zn}-\mathrm{SOD}$ was downregulated in the STZ rats, compared to that in the Con rats (Figure 5). PCA treatment significantly enhanced the protein expression of $\mathrm{Cu} / \mathrm{Zn}-\mathrm{SOD}$. Furthermore, markedly reduced vascular total SOD activity was observed in the STZ group, and PCA treatment ameliorated this decrease (Figure 6B).

\section{Discussion}

Intraperitoneal injection of STZ induced a significant elevation of plasma glucose levels in rats, which was associated with the impairment of endothelium-dependent relaxation. Treatment with PCA for 6 weeks significantly ameliorated the impairment of acetylcholine-evoked 
A

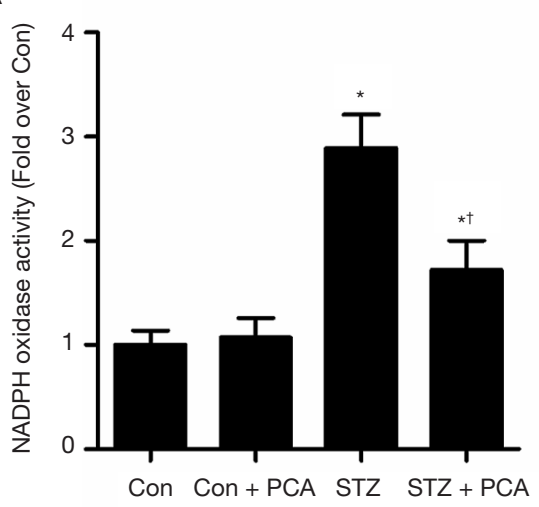

B

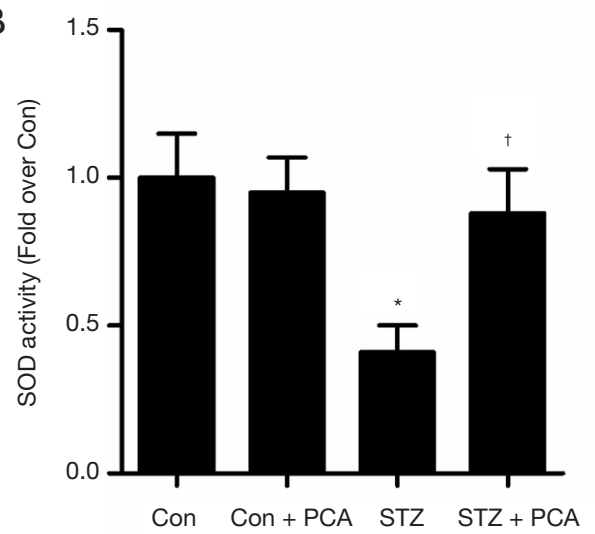

Figure 6 Effects of protocatechualdehyde (PCA) on activity of NADPH oxidase (A) and total SOD (B). Values are displayed as mean \pm SD, $\mathrm{n}=4-5$ per group. ${ }^{*} \mathrm{P}<0.05$ vs. Con rats; ${ }^{\dagger} \mathrm{P}<0.05$ vs. streptozotocin $(\mathrm{STZ})$ rats.

endothelium-dependent relaxation. This beneficial effect is evidently unrelated to the correction of hyperglycemia, because PCA treatment did not significantly affect blood glucose content in DM rats.

The NOS-produced signaling molecule NO has vital involvement in numerous physiological processes, such as vasodilation, neuronal transmission, and immune responses $(19,20)$. A reduction in NO bioactivity is the most typical characteristic of endothelial dysfunction, and is mainly attributable to an eNOS-induced decrease in NO synthesis, as well as an increase in superoxide anion-induced NO oxidative inactivation $(13,17)$. Consistent with the findings of previous studies $(4,21)$, this study found that the levels of aortic P-eNOS/T-eNOS expression and eNOS activity were decreased in STZ rats. However, PCA treatment had no effect on P-eNOS expression or eNOS activity, indicating that the improvement of aortic NO bioactivity cannot be attributed to endothelial NO synthesis.

Superoxide anion, one of the most important vasoactive oxygen species, reacts with NO to produce peroxynitrite, which results in $\mathrm{NO}$ oxidative inactivation (7). In the present study, the STZ rats displayed increased vascular superoxide anion production and an elevated level of aortic nitrotyrosine. The decrease in acetylcholineinduced relaxation in the STZ-induced diabetic aorta was significantly rescued by incubation with MPG. However, NO oxidative inactivation reflected the difference between ACh-induced maximum relaxation values calculated with and without the presence of MPG (4), and this value was lower in PCA-treated rats than in STZ rats. PCA has been previously demonstrated to decrease the DNA oxidation biomarker 8-hydroxygluanine in human lens epithelial cells following incubation with methylglyoxal (11). Furthermore, the aortas of PCA-treated diabetic rats displayed decreased superoxide anion generation and nitrotyrosine levels. These data indicate that the improvement in $\mathrm{NO}$ oxidative inactivation may be the main reason for the rise in aortic NO bioactivity observed after PCA treatment.

Superoxide anion is produced by NADPH oxidase and eliminated by endogenous antioxidant SOD. NADPHoxidases are considered to be the most important source of superoxide anion in the vessel wall $(13,14)$. Cytosolic $\mathrm{Cu} /$ $\mathrm{Zn}-\mathrm{SOD}$, mitochondrial Mn-SOD and extracellular SOD enzymes are involved in the scavenging of superoxide anion and play important roles in balancing ROS generation. The total SOD activity and extracellular $\mathrm{Cu} / \mathrm{Zn}$-SOD expression were reduced in the aorta of long-term diabetic rats (22). In the present study, elevated aortic $\mathrm{Cu} / \mathrm{Zn}$-SOD expression and SOD activity, along with decreases in NADPH oxidase subunits $\mathrm{p} 22^{\text {phox }}$ and $\mathrm{p} 47^{\text {phox }}$ expression and NADPH oxidase activity, were observed in diabetic rats following PCA treatment. Therefore, by protecting aortic endothelium against oxidative stress, PCA may avert the breakdown of NO, thus improving vascular function.

In conclusion, our results suggest that PCA has the protective effects against oxidative stress and restores endothelial function in STZ-diabetic rats. These effects are attributable, at least in part, to an improvement in vascular $\mathrm{NO}$ oxidative inactivation induced by PCA. These findings indicate that PCA may be useful to improve vascular function in patients with DM. Nonetheless, it is noteworthy that our findings were mainly based on rodent models. 


\section{Page 8 of 9}

Therefore, further study is needed to evaluate the effects of PCA on vascular function in patients with DM.

\section{Acknowledgments}

The authors appreciate the academic support from AME Diabetes Collaborative Group.

Funding: The current study was supported by a grant from the Science and Technology Bureau of Wenzhou (grant No. Y20170646).

\section{Footnote}

Reporting Checklist: The authors have completed the ARRIVE reporting checklist. Available at http://dx.doi. org/10.21037/atm-21-1431

Data Sharing Statement: Available at http://dx.doi. org/10.21037/atm-21-1431

Conflicts of Interest: All authors have completed the ICMJE uniform disclosure form (available at http://dx.doi. org/10.21037/atm-21-1431). The authors have no conflicts of interest to declare.

Ethical Statement: The authors are accountable for all aspects of the work in ensuring that questions related to the accuracy or integrity of any part of the work are appropriately investigated and resolved. Experiments were performed under a project license (No.: 201700103) granted by the Wenzhou Medical College Animal Policy and Welfare Committee. All animal experiments complied with the Wenzhou Medical College Guidelines on the Care and Use of Laboratory Animals.

Open Access Statement: This is an Open Access article distributed in accordance with the Creative Commons Attribution-NonCommercial-NoDerivs 4.0 International License (CC BY-NC-ND 4.0), which permits the noncommercial replication and distribution of the article with the strict proviso that no changes or edits are made and the original work is properly cited (including links to both the formal publication through the relevant DOI and the license). See: https://creativecommons.org/licenses/by-nc-nd/4.0/.

\section{References}

1 Bigagli E, Lodovici M. Circulating Oxidative Stress
Ji et al. Protocatechualdehyde improves endothelial dysfunction

Biomarkers in Clinical Studies on Type 2 Diabetes and its Complications. Oxid Med Cell Longev 2019;2019:5953685.

2 Andary R, Fan W, Wong ND. Control of cardiovascular risk factors among US adults with type 2 diabetes with and without cardiovascular disease. Am J Cardiol 2019;124:522-7.

3 Mori H, Okada Y, Kawaguchi M, et al. A study of the vascular endothelial function in patients with type 2 diabetes mellitus and rheumatoid arthritis. Intern Med 2019;58:1383-90.

4 Matsumoto T, Noguchi E, Kobayashi T, et al. Mechanisms underlying the chronic pioglitazone treatment-induced improvement in the impaired endothelium-dependent relaxation seen in aortas from diabetic rats. Free Radic Biol Med 2007;42:993-1007.

5 Koyama T, Tanaka A, Yoshida H, et al. Comparison of the effects of linagliptin and voglibose on endothelial function in patients with type 2 diabetes and coronary artery disease: A prospective, randomized, pilot study (EFFORT). Heart Vessels 2018;33:958-64.

6 Sun HJ, Chen D, Wang PY, et al. Salusin- $\beta$ Is Involved in Diabetes Mellitus-Induced Endothelial Dysfunction via Degradation of Peroxisome ProliferatorActivated Receptor Gamma. Oxid Med Cell Longev 2017;2017:6905217.

7 Majithiya JB, Paramar AN, Balaraman R. Pioglitazone, a PPARgamma agonist, restores endothelial function in aorta of streptozotocin-induced diabetic rats. Cardiovasc Res 2005;66:150-61.

8 Hirata M, Serizawa K, Aizawa K, et al. 22-Oxacalcitriol prevents progression of endothelial dysfunction through antioxidative effects in rats with type 2 diabetes and early-stage nephropathy. Nephrol Dial Transplant 2013;28:1166-74.

9 Privistirescu AI, Sima A, Duicu OM, et al. Methylene blue alleviates endothelial dysfunction and reduces oxidative stress in aortas from diabetic rats. Can J Physiol Pharmacol 2018;96:1012-16.

10 Kim YS, Kim NH, Lee SW, et al. Effect of protocatechualdehyde on receptor for advanced glycation end products and TGF-beta1 expression in human lens epithelial cells cultured under diabetic conditions and on lens opacity in streptozotocin-diabetic rats. Eur J Pharmacol 2007;569:171-9.

11 Guo C, Wang S, Duan J, et al.

Protocatechualdehyde protects against cerebral ischemiareperfusion-induced oxidative injury via protein 
kinase cepsilon/Nrf2/HO-1 pathway. Mol Neurobiol 2017;54:833-45.

12 Wang YH, Han YP, Yu HT, et al. Protocatechualdehyde prevents methylglyoxal-induced mitochondrial dysfunction and AGEs-RAGE axis activation in human lens epithelial cells. Eur J Pharmacol 2014;738:374-83.

13 Kong X, Li W, Guo L, et al. Sesamin enhances nitric oxide bioactivity in aortas of spontaneously hypertensive rats. Ther Adv Cardiovasc Dis 2015;9:314-24.

14 Kong X, Yang JR, Guo LQ, et al. Sesamin improves endothelial dysfunction in renovascular hypertensive rats fed with a high-fat, high-sucrose diet. Eur J Pharmacol 2009;620:84-9.

15 Ulker S, McMaster D, McKeown PP, et al. Impaired activities of antioxidant enzymes elicit endothelial dysfunction in spontaneous hypertensive rats despite enhanced vascular nitric oxide generation. Cardiovasc Res 2003;59:488-500.

16 Sun X, Jiao X, Ma Y, et al. Trimethylamine N-oxide induces inflammation and endothelial dysfunction in human umbilical vein endothelial cells via activating ROSTXNIP-NLRP3 inflammasome. Biochem Biophys Res Commun 2016;481:63-70.

17 Kong X, Ma MZ, Qin L, et al. Pioglitazone enhances the blood pressure-lowering effect of losartan via synergistic

Cite this article as: Ji B, Yuan K, Li J, Ku BJ, Leung PS, He W. Protocatechualdehyde restores endothelial dysfunction in streptozotocin-induced diabetic rats. Ann Transl Med 2021;9(8):711. doi: 10.21037/atm-21-1431 attenuation of angiotensin II-induced vasoconstriction. J Renin Angiotensin Aldosterone Syst 2014;15:259-70.

18 Papparella I, Ceolotto G, Montemurro D, et al. Green tea attenuates angiotensin II-induced cardiac hypertrophy in rats by modulating reactive oxygen species production and the Src/epidermal growth factor receptor/Akt signaling pathway. J Nutr 2008;138:1596-601.

19 Lee SH, Park CS, Ok SH, et al. Bupivacaine-induced contraction is attenuated by endothelial nitric oxide release modulated by activation of both stimulatory and inhibitory phosphorylation (Ser1177 and Thr495) of endothelial nitric oxide synthase. Eur J Pharmacol 2019;853:121-8.

20 Hariharan A, Jing Y, Collie ND, et al. Altered neurovascular coupling and brain arginine metabolism in endothelial nitric oxide synthase deficient mice. Nitric Oxide 2019;87:60-72.

21 Roghani-Dehkordi F, Roghani M, Baluchnejadmojarad T. Diosgenin mitigates streptozotocin diabetes-induced vascular dysfunction of the rat aorta: The involved mechanisms. J Cardiovasc Pharmacol 2015;66:584-92.

22 Matsumoto T, Noguchi E, Kobayashi T, et al. Mechanisms underlying the chronic pioglitazone treatment-induced improvement in the impaired endothelium-dependent relaxation seen in aortas from diabetic rats. Free Radic Biol Med 2007;42:993-1007. 